\title{
Sensorless force control for dielectric elastomer transducers
}

Journal of Intelligent Material System and Structures 2019, Vol. 30(9) 1419-1434 (C) The Author(s) 2018 Article reuse guidelines: sagepub.com/journals-permissions DOI: 10.1 I77/I045389XI7754255 journals.sagepub.com/home/jim (SAGE

\author{
Thorben Hoffstadt $\mathbb{D}$ and Jürgen Maas
}

\begin{abstract}
Multilayer stack-transducers made of dielectric elastomers generate considerable tensile forces and deformations when they are electrically stimulated. Thus, due to their capacitive behavior, they are for example energy efficient substitutes for conventional electromagnetic drives and enable various further completely new applications. Within this contribution, we present a sensorless force control for dielectric elastomer stack-transducers driven by a bidirectional flybackconverter. This force control for example can be used as interface for superimposed application-oriented controls. First of all, the properties of dielectric elastomer stack-transducers and the flyback-converter as well as its control structure are summarized. Due to the characteristic behavior of the power converter, the design of a sliding mode force controller is carried out based on an analytical model of the coupled electromechanical system containing the transducer and driving electronics. Using this model, the transducer force can be determined with the measured driving voltage and deformation so that an explicit force measurement is not required. In a first step, a two-point controller is developed for the variable structure control that is afterward extended in order to improve the control quality, for example, by adapting the inner power converter control. Finally, the experimental validations with a prototype stack-transducer and bidirectional flyback-converter demonstrate the feasibility as well as the precision and dynamics of the developed force control.
\end{abstract}

\section{Keywords}

dielectric elastomer transducers, force control, sensorless control

\section{Introduction}

Smart materials offer various opportunities to realize scalable, efficient, and easy to integrate transducers. They have unique mechanical as well as electrical properties that enable advantageous features for a wide range of applications as actuators, sensors, and generators. Hence, beside completely new applications, these transducers are often superior substitutes for conventional electromechanical actuators, with extensively improved power consumption, force density, and flexibility. However, depending on the kind of application, the transducers are part of a control loop, so that a certain control interface has to be provided. For this purpose, Maas and Soetebier (2012) have proposed a force control interface realized under consideration of the utilized smart material and its driving power electronics. With this interface, the novel transducer system can be integrated in a superimposed application-oriented control loop, for example, a motion or impedance control.

Within this contribution, transducers based on dielectric elastomer (DE) are considered consisting of a hyperelastic, incompressible elastomer coated with compliant electrodes on its surfaces. By applying an electric field strength $E$ to the DE, the resulting electrostatic pressure $\sigma_{e l}$ depending on the material's permittivity $\varepsilon=\varepsilon_{0} \cdot \varepsilon_{r}$ describes the electromechanical coupling

$$
\sigma_{e l}=\varepsilon_{0} \cdot \varepsilon_{r} \cdot E^{2}=\varepsilon_{0} \cdot \varepsilon_{r} \cdot \frac{v_{p}^{2}}{d^{2}}=\frac{2}{V_{e}} \cdot U_{c, \text { diel }}
$$

It also can be expressed by the energy $U_{c \text {, diel }}$ stored in the DE capacitance obtained by the elastomer material covered with electrode resulting in the active volume $V_{e}$.

Based on this, electromechanical coupling actuators (Giousouf and Kovacs, 2013; Price and Ask, 2014), generators (Maas and Graf, 2012; Pelrine et al., 2001) as well as sensors (Anderson et al., 2012; Hoffstadt

Mechatronic Systems Laboratory, Faculty V - Mechanical Engineering and Transport Systems, Technische Universität Berlin, Berlin, Germany

\section{Corresponding author:}

Thorben Hoffstadt, Mechatronic Systems Laboratory, Faculty V Mechanical Engineering and Transport Systems, Technische Universität Berlin, Hardenbergstrasse 36, 10623 Berlin, Germany.

Email: thorben.hoffstadt@emk.tu-berlin.de 
et al., 2014) can be realized and were investigated in several publications, see for example Rosset et al. (2016), Hodgins et al. (2013), and Zhang et al. (2006). Since very thin polymer films with thickness $d$ are used to achieve high electric field strength $E$ with considerable low voltages $v_{p}$, multilayer technologies are meaningful for $\mathrm{DE}$ actuators to either increase the deformation or force of the actuator. Thus, these actuators are predestined among other for positioning applications like in pneumatic valves or electrical switches, see Price and Ask (2014), or for haptic feedback applications, for example, as proposed by Biggs et al. (2013).

For different DE transducer topologies and applications, control concepts have been published previously. Kaal and Herold (2011) have compensated the nonlinear static voltage-strain behavior of a DE actuator by implementing an inverted feed-forward structure to obtain a linear static behavior. This scheme was used to realize an active vibration control. However, by this approach, only the static behavior can be compensated. In the higher frequency range, a sufficient control quality is only obtained when the viscous material properties are almost negligible.

For a circular single-layer DE actuator, Dubois et al. (2008) propose a model-based approach to control the resonance frequency of the DE membrane. As a possible application of such a device, also a tunable attenuator is mentioned. Sarban and Jones (2012) developed a model-based feed-forward control for a DE roll-actuator used for active vibration damping. In contrast, Zou et al. (2016) present a feed-forward control for the creep compensation of a membrane actuator, while $\mathrm{Gu}$ et al. (2015) use a comparable structure for the open-loop deformation control of a planar DE actuator.

In addition, position control designs have been carried out using the aforementioned static linearization by implementing a square-root function in the forward path. In particular, Rizzello et al. (2016) designed a proportional-integral-derivative (PID) controller for a DE membrane actuator, while Jones and Sarban (2012) developed an internal model position control for a DE roll-actuator. The control scheme suggested by Wilson et al. (2016) uses a cerebellar-based adaptive-inverse model control especially to improve the behavior in case of DE materials with dominant creep.

However, due to the very fast and powerful laboratory high-voltage amplifiers used within the aforementioned publications, it was sufficient to neglect the dynamics of this amplifier when designing the control, although silicone-based DE materials with very low viscoelastic time constant have been used. Furthermore, the referenced controllers were realized within one single control loop. In contrast, it is also possible to establish an inner force control interface. Subsequently, comparable to conventional drive systems, this control interface can be used to realize the application-oriented control within superimposed control loops as already mentioned above. Force control interfaces are also very common in terms of the control of (soft) robotics, for example, for rehabilitation devices (Maciejasz et al., 2014) or artificial muscles (Tomori et al., 2013) under particular consideration of the utilized actuator principles.

In the conference proceedings (Hoffstadt and Maas, 2015b), an open-loop force control for a DE stackactuator was proposed that calculates the required terminal voltage to obtain the set-point force. Based on a simplified linearelastic model of the DE stack-actuator and the measured terminal voltage, the elastic deformation as well as viscous material tensions was estimated and compensated by a feed-forward control. Using this estimation, a measurement of the actuator force, that is, the control variable, was not necessary.

Within this contribution, this approach is extended to a sensorless closed-loop force control taking into account the nonlinear DE stack-actuator dynamics as well as the characteristics of the feeding power electronics. The utilized bidirectional flyback-converter supplies an almost constant but adaptable feeding power over the whole operating range. While DE stackactuators can be modeled with similar approaches as used for other topologies, for example, a membrane actuator, the mentioned converter behaves completely different than the usually used high-voltage amplifiers. Due to this, a PID controller as used by Rizzello et al. (2016) and Chuc et al. (2011) is not sufficient if high dynamics and accuracy should be obtained. The strongly nonlinear behavior of the control plant with the flyback-converter and the DE stack-actuator requires a particular control strategy to ensure high dynamics, robustness, and sufficient accuracy. Thus, here a sliding mode controller (SMC) according to DeCarlo et al. (1988) is designed for the intended force control. To our best knowledge, it is the first time that both the properties of the DE actuator and the predestined power electronics are taken into account for the design of the transducer control.

In the following section, first of all, the considered system comprising a DE stack-transducer and a bidirectional flyback-converter is introduced and an electromechanically coupled model (Hoffstadt and Maas, 2015a) for their dynamics is presented. One of the state variables in this model is assigned to the electrical field energy in the elastomer capacitance as an equivalent quantity. This energy results in the electrostatic pressure, see equation (1), and thus causes the actuator force. Hence, by controlling the energy, the force is controlled, too. The design of the sliding mode force control or corresponding energy control is carried out based on the holistic electromechanical model, afterward. In this content, the focus is also given on the optimization of the control with respect to minimized losses by reducing the switching frequency of the 


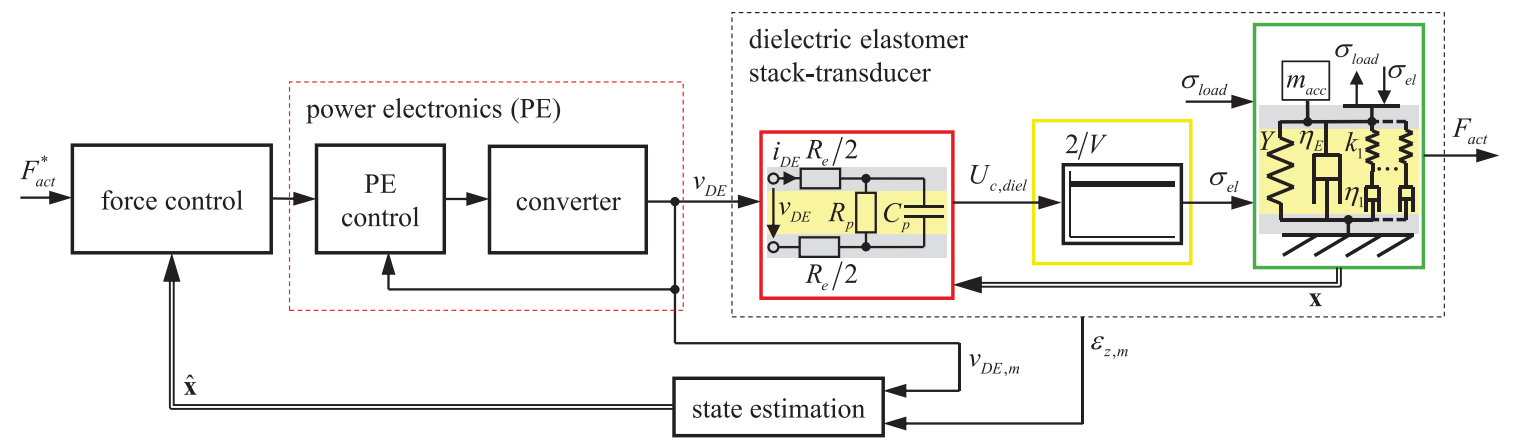

Figure I. Structure of the sensorless closed-loop force control for DE stack-transducers fed by a bidirectional power electronics.

flyback-converter as well as an improved control accuracy by adapting the inner control of the power converter.

Due to the nonlinear system dynamics, an extended Kalman filter (EKF) is used here to estimate all required states for the control based on the measured terminal voltage and the deformation of the stacktransducer. Overall, this results in the structure of the closed-loop force control schematically depicted in Figure 1. Finally, the obtained force control is experimentally validated using an in-house developed prototype of the converter and an automatically manufactured prototype of the DE stack-transducer.

\section{Overall system model for DE stack- transducers driven by bidirectional flyback-converters}

Before the design of the proposed force control is carried out, first of all the utilized DE stack-transducer and driving power electronics are briefly introduced. Both parts result in an electromechanically coupled model of the overall system that is afterward used for the control design.

\section{Multilayer DE stack-transducer}

The design and fabrication of the considered multilayer DE stack-transducers are described in detail by Maas et al. (2015). In general, this transducer consists of $N$ single DE actuator films with initial thickness $d_{0}$ stacked on top of each other. Hence, these films are mechanically connected in series while the electrical supply is ensured via a connection in parallel. Centrally on the DE transducer films, electrodes with area $A_{e}=w_{e} \cdot l_{e}$ are applied. In order to prevent electrical arcing, this area is surrounded by an insulating area, that is, the transducer films have a total cross-sectional area of $A_{0}=l_{0} \cdot w_{0}$, see Figure 2. By stacking $N$ films, the active volume $V_{e}=A_{e} \cdot N \cdot d_{0}$ and overall transducer volume $V=A_{0} \cdot N \cdot d_{0}$ can be distinguished.
Due to the mechanical connection in series, the deformations of the single films sum up, while the transducer force is scaled by the active area $A_{e}$ of the stacked transducer films. Considering a quasi-static operation, a stack-actuator generates a tensile force $F_{a c t}$ depending on the electrostatic pressure $\sigma_{e l}$ applied on the active area $A_{e}$ and the elastic deformation tension $\sigma_{\text {elast }}$ on the overall area $A$

$$
F_{a c t}=A \cdot \sigma_{a c t}=A_{e} \cdot \sigma_{e l}-A \cdot \sigma_{\text {elast }}
$$

This tensile force can be transmitted if mechanical interfaces are applied on the top and bottom layers of the stack-actuator for the integration into the intended application, see Figure 3(a). Since a part of the electrostatic pressure is used to elastically deform the actuator, the actuator force is decreased with increasing deformation as can be seen in the strain-force characteristics in Figure 3(b). However, adapting the electrostatic pressure $\sigma_{e l}$ by the applied electrical field strength $E_{0}=v_{p} / d_{0}$, the force $F_{a c t}$ can be adjusted in a certain range for a given deformation. Assuming an almost homogeneous deformation, these characteristics can be modeled analytically as subsequently explained and experimentally validated for the use of the intended force control, while the dashed lines in Figure 3(b) represent results of a finite element analysis (FEA) under consideration of constraints due to the actuator design published by Kuhring et al. (2015).

\section{Bidirectional flyback-converter}

From an electrical point of view, a DE transducer represents a capacitive load, given by the DE films electrically connected in parallel, with additional resistances in series and parallel to take into account the non-ideal behavior of the utilized electrode and the dielectric loss, respectively. Thus, in order to control the voltage across the DE, a controlled current source is required as power electronics, see Maas and Graf (2011). Among others, for moderate output powers, a 


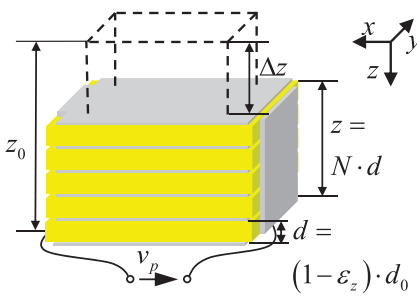

(a)

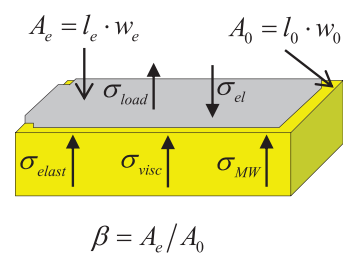

(b)
Figure 2. Schematic design and dimensions of the considered DE stack-transducer (a) and relevant tensions to model the strain-force behavior (b).

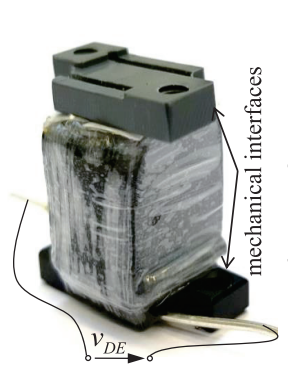

(a)

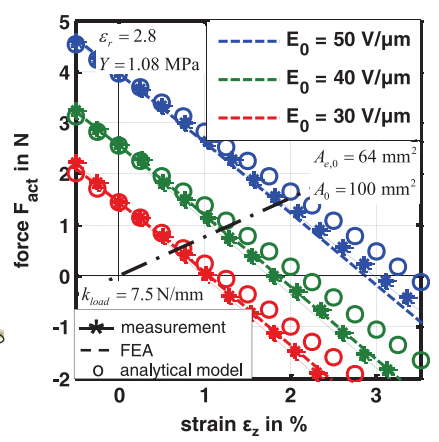

(b)
Figure 3. Prototype of a silicone-based DE stack-transducer with two interfaces (a) and its strain-force characteristics (b).

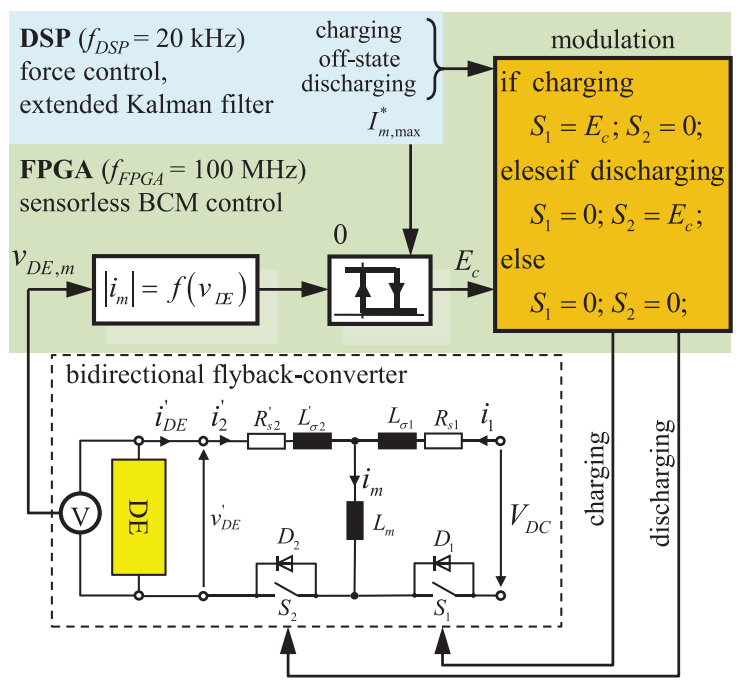

Figure 4. Bidirectional flyback-converter with sensorless hysteresis current control operating the driving electronics in boundary conduction mode (BCM).

flyback-converter as schematically depicted in Figure 4 provides this capability with output voltages in the kilovolt-range. Furthermore, a bidirectional energy flow can be enabled by including switches $S_{1}$ and $S_{2}$ on the primary and secondary side, respectively. For charging a DE transducer solely $S_{1}$ is modulated while $S_{2}$ is opened. Vice versa, for discharging $S_{2}$ is used, see Hoffstadt and Maas (2016).

Here, the magnetizing inductance $L_{m}$ of the flyback transformer is used as an energy storage element analog to the inductor in a buck-boost converter (Venkatesan, 1989). By adjusting a certain peak value $I_{m, \max }^{*}$ of the magnetizing current $i_{m}=i_{1}+i_{2}^{\prime}$, the energy increment $\Delta U_{\max }$ stored in $L_{m}$ is given by

$$
\Delta U_{\max }=\frac{1}{2} \cdot L_{m} \cdot I_{m, \max }^{* 2}
$$

This characteristic behavior results in a mean output power $\bar{p}$ per switching period $T_{S}$ given by three discrete states

$$
\bar{p}=\left\{\begin{array}{cc}
+\bar{p}_{\max }=+\frac{\Delta U_{\max }}{T_{S}}, \quad \text { charging } \\
0, \quad \text { off }- \text { state } \\
-\bar{p}_{\max }=-\frac{\Delta U_{\max },}{T_{S}}, \quad \text { discharging }
\end{array}\right.
$$

As shown by Sokal and Redl (1997), this energy increment is transferred from primary to secondary side or vice versa in the shortest possible time $T_{S}$ if the flyback-converter is operated in boundary conduction mode (BCM). In case of the BCM, the magnetizing current $i_{m}$ alternates between zero and its maximum value $I_{m, \max }^{*}$. The next switching period starts right in the moment when $i_{m}$ becomes zero. Therefore, to operate the flyback-converter in this BCM sensors are required to detect whether the magnetizing current has reached these thresholds. Then the triggering signal $E_{c}$ can be generated for the primary and secondary switches $S_{1}$ and $S_{2}$, respectively. Beside measuring the current for this purpose, a sensorless hysteresis current control based on the model of the bidirectional flybackconverter depicted in the dashed box in Figure 4 and the measured output voltage $v_{D E}$ of the DE transducer can be implemented, as proposed in Hoffstadt and Maas (2016). This sensorless current control solely requires a measurement of the output voltage but not of the current, that is, the measurement effort can be significantly reduced.

Using this control with a superimposed voltage control loop, the measurement results in Figure 5 are obtained with a prototype of the bidirectional flybackconverter. As can be seen in the two plots in the middle showing the magnetizing current $i_{m}=i_{1}+i_{2}^{\prime}$, the switching period $T_{S}$ in BCM decreases with increasing output voltage $v_{D E}$. However, as $T_{S}$ only changes significantly for voltages below $500 \mathrm{~V}$, the mean output power $\bar{p}$ in equation (4) is almost constant for a chosen $I_{m, \max }^{*}$ during charging and discharging, as can be seen in the bottom plot. This results in the characteristic change in voltage that approximately can be described by a square-root function for a capacitive load 

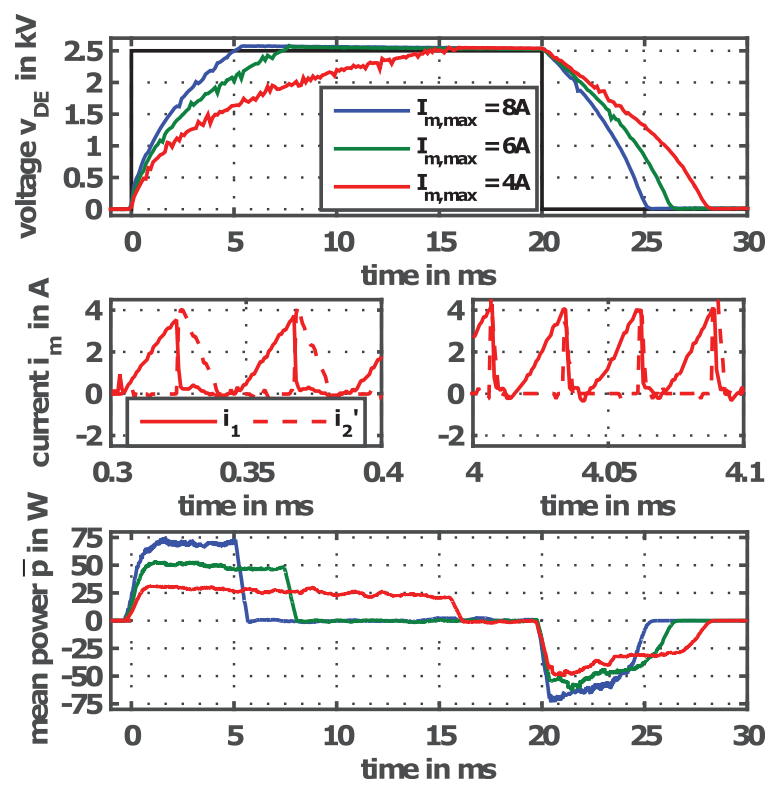

Figure 5. Output voltage $v_{D E}$, sections of the magnetizing current $i_{D E}=i_{1}+i_{2}^{\prime}$, and mean power $\bar{p}$ obtained when feeding a DE capacitance of $C_{p}=100 \mathrm{nF}$ with the bidirectional flybackconverter using the BCM control.

(Hoffstadt and Maas, 2016). Furthermore, by varying $I_{m, \text { max }}^{*}$ according to equation (3), the feeding power in equation (4) can be continuously adjusted, too. In case of a smaller $I_{m \text {, max }}^{*}$, it takes longer to charge the capacitance of $C_{p, 0}=100 \mathrm{nF}$ to $2.5 \mathrm{kV}$. By reducing $I_{m, \max }^{*}$, the resolution of the sliding mode control can be increased. As explained in the control section in detail, this will be used for the adaptation of the control.

\section{Holistic transducer model}

The subsequent design of the sensorless force control is based on a holistic model of the DE stack-transducer that was published previously (Hoffstadt and Maas, 2015a) and is illustrated in Figure 6. While in the previous publication, the stretch $\lambda_{z}$, defined as the ratio of the actual transducer height $z$ to its initial value $z_{0}=N \cdot d_{0}$, was used to describe the deformation state; here, the strain $\varepsilon_{z}=\Delta z / z_{0}$ in positive $z$-direction of actuation is considered (see Figure 2) depending on the compression $\Delta z$ because this quantity is more common in control engineering. Nevertheless, the stretch and strain can be related as follows

$$
\lambda_{z}=\frac{z}{z_{0}}=\frac{z_{0}-\Delta z}{z_{0}}=1-\frac{\Delta z}{z_{0}}=1-\varepsilon_{z}
$$

As already depicted in Figure 1, the model of a DE transducer comprises a mechanical (green box) and an electrical domain (red box) that influence each other due to the electromechanical coupling (yellow box). Within the mechanical domain, the nonlinear viscoelastic behavior is modeled by a generalized Kelvin-Maxwell model with the corresponding tensions already depicted in Figure 2(b). Depending on the applied electrostatic pressure $\sigma_{e l}$ and an external load tension $\sigma_{\text {load }}$, the strain $\varepsilon_{z}$ is obtained. In case of strains smaller than $20 \%$, the Neo-Hookean approach is sufficient to model the quasi-static hyperelastic material behavior (Hoffstadt and Maas, 2015a). Under consideration of the DE stack-transducer design in Figure 2 , the almost equi-biaxial in-plane deformation is given by

$$
\sigma_{\text {elast }}=\frac{Y}{3} \cdot\left(\frac{1}{1-\varepsilon_{z}}-\left(1-\varepsilon_{z}\right)^{2}\right)
$$

depending on the material's Young's modulus $Y$. Viscoelasticity is considered by a linear viscosity $\eta_{E}$ resulting in

$$
\sigma_{v i s c}=\eta_{E} \cdot \dot{\varepsilon}_{z}
$$

and one additional Maxwell element. In contrast to the hyperelastic behavior depending on the strain $\varepsilon_{z}$, the viscoelastic tension of the Maxwell element is determined with the strain $\varepsilon_{k 1}$ of its spring $k_{1}$ since it is assumed to be linear and connected in series to the dashpot $\eta_{1}$

$$
\dot{\varepsilon}_{k 1}=\dot{\varepsilon}_{z}-\frac{k_{1}}{\eta_{1}} \cdot \varepsilon_{k 1} \rightarrow \sigma_{M W}=k_{1} \cdot \varepsilon_{k 1}
$$

Hence, the overall actuator tension can be modeled by

$$
\sigma_{a c t}=\beta \cdot \sigma_{e l}-\sigma_{\text {elast }}-\sigma_{\text {visc }}-\sigma_{M W}
$$

Here, $\beta=A_{e} / A$ takes into account that the electrostatic pressure $\sigma_{e l}$ only acts on the area $A_{e}$ covered with electrode, compare equation (2). Under consideration of equation (1), the effective electrostatic pressure can be expressed with the overall transducer volume $V$ by

$$
\begin{aligned}
& \beta \cdot \sigma_{e l}=\frac{A_{e}}{A} \cdot \frac{2}{V_{e}} \cdot U_{c, \text { diel }}=\frac{2}{V} \cdot U_{c, \text { diel }} \\
& \text { with } V_{e}=A_{e} \cdot z \text { and } V=A \cdot z
\end{aligned}
$$

The actuator tension $\sigma_{a c t}$ works against an external load tension $\sigma_{\text {load }}$ and results in the acceleration in direction of actuation

$$
z_{0} \cdot \ddot{\varepsilon}_{z}=\frac{A}{m_{\text {acc }}} \cdot\left(\sigma_{\text {act }}-\sigma_{\text {load }}\right)=\frac{A_{0} \cdot\left(\sigma_{\text {act }}-\sigma_{\text {load }}\right)}{m_{\text {acc }} \cdot\left(1-\varepsilon_{z}\right)}
$$

considering equi-biaxial in-plane extension for the incompressible elastomer material by

$$
A=\frac{A_{0}}{1-\varepsilon_{z}}
$$

Here, $m_{a c c}$ comprises the accelerated actuator mass as well as the additional mass $m_{\text {add }}$ of components 


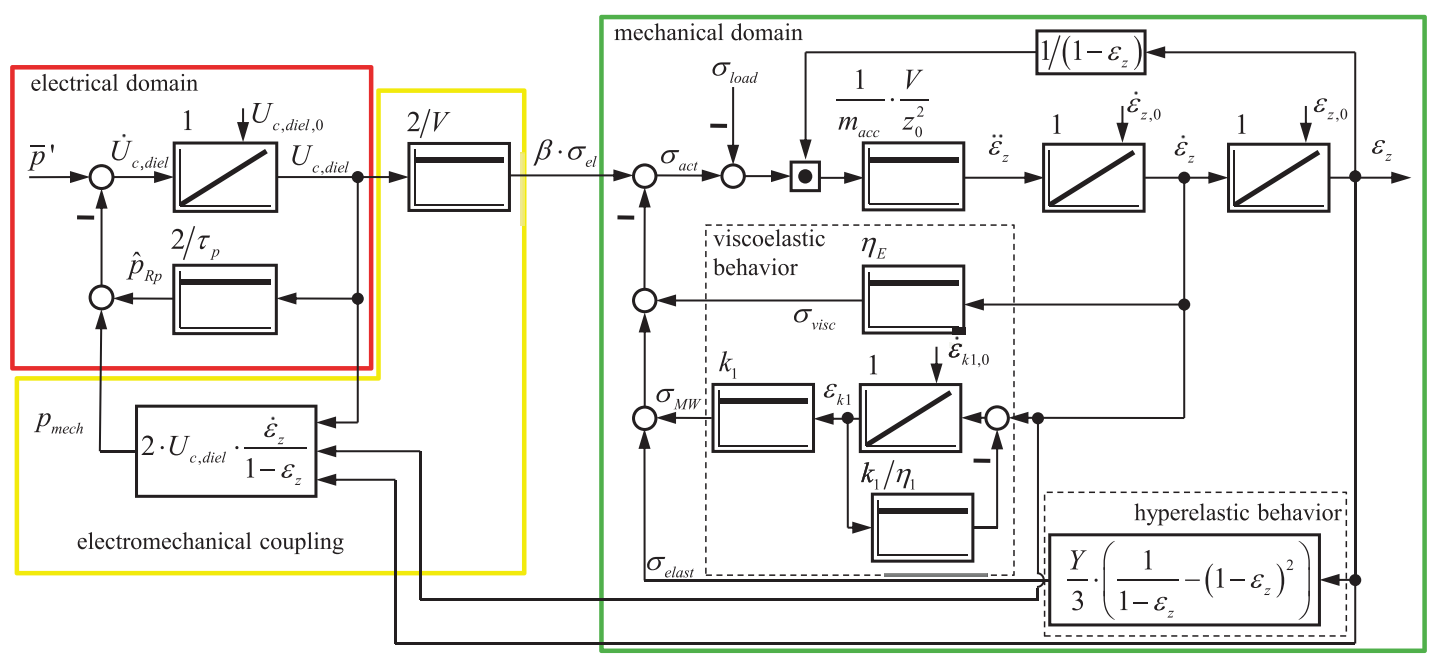

Figure 6. Holistic model of a DE stack-transducer driven by an averaged input power $\bar{p}^{\prime}$ supplied for example by the bidirectional flyback-converter.

attached to the stack-actuator. According to Piersol and Paez (2010), a third of the continuously distributed transducer mass $m_{D E}$ is accelerated, resulting in

$$
m_{a c c}=\frac{m_{D E}}{3}+m_{a d d}
$$

This finally yields the nonlinear action diagram in Figure 6. As already mentioned above, the functional principle of a DE actuator is given by the electrostatic pressure $\sigma_{e l}$ in equation (1). Thus, the energy $U_{c, \text { diel }}$ as an output quantity of the electrical domain acts on the mechanical domain by a linear relation. Although this energy is stored in the active volume depending on the area $A_{e}$ covered with electrode, the overall volume $V$ of the DE stack-actuator is used here in order to take into account that the electrostatic pressure has to deform the whole actuator with its insulating and, thus, passive volumes.

The electrical domain is derived based on a consideration of a power balance. This balance is obtained under consideration of the electrical equivalent circuit in Figure 1 (red box). The model comprises a series resistance $R_{e}$ representing losses of the electrode, a parallel resistance $R_{p}$ of the non-ideally insulating polymer, and its capacitance $C_{p}$. Due to the deformation under actuation, the values of these parameters depend on the stretch.

While Graf and Maas (2011) have derived analytical expressions for the stretch dependency of the resistance $R_{p}$ and the capacitance $C_{p}$, a suitable relation for the electrode resistance is difficult to find. The behavior of the electrode strongly depends on the utilized electrode material and its structure. However, concerning the intended power balance, the electrode causes losses $\bar{p}_{R_{e}}$ during charging and discharging operation but not during the off-state of the utilized converter. Thus, for the further considerations, the output power $\bar{p}$ in equation (4) is reduced by the losses $\bar{p}_{R_{e}}$ resulting in the mean feeding power

$$
\bar{p}^{\prime}=\bar{p}-\bar{p}_{R_{e}}
$$

The loss $\bar{p}_{R_{e}}=R_{e} \cdot i_{D E}^{2}$ depends on the resistance $R_{e}$ and the feeding current $i_{D E}$. As the current is discontinuous and depending on the charging state, it is hard to find an analytic approximation for this loss. However, for the intended SMC, it is not necessary to exactly determine this loss. As shown in the following section, it has to be distinguished between charging, discharging, and off-state, so that $\bar{p}^{\prime}$ can be used as control input. In addition, the parallel resistance $R_{p}$ causes losses $p_{R_{p}}$, too. A part of the power is converted into mechanical power $p_{\text {mech }}$ that deforms the DE and works against the load $\sigma_{\text {load }}$. Under consideration of all of these quantities, the power balance can be derived by

$$
\dot{U}_{c, \text { diel }}=\bar{p}^{\prime}-p_{R_{p}}-p_{\text {mech }} \text {, with } p_{\text {mech }}=p_{\text {act }}+p_{\text {kin }}
$$

Here, the losses $p_{R_{p}}$ are obtained by taking into account the parallel connection of the DE capacitance $C_{p}$ and the resistance $R_{p}$ that are obtained by connecting the $N$ single DE transducer films in parallel. By defining the time constant $\tau_{p}$, the losses linearly depend on the energy $U_{c, \text { diel }}$

$$
\begin{gathered}
\bar{p}_{R p}=\frac{v_{p}^{2}}{R_{p}}=\frac{2 \cdot U_{c, \text { diel }}}{C_{p} \cdot R_{p}}=\frac{2 \cdot U_{c, \text { diel }}}{\tau_{p}} \\
\text { with } U_{c, \text { diel }}=\frac{C_{p}}{2} \cdot v_{p}^{2} \\
\text { and } \tau_{p}=\underbrace{N \cdot \varepsilon_{0} \cdot \varepsilon_{r} \cdot \frac{A}{d}}_{C_{p}} \cdot \underbrace{\frac{1}{N} \cdot \rho_{p} \cdot \frac{d}{A}}_{R_{p}}=\varepsilon_{0} \cdot \varepsilon_{r} \cdot \rho_{p}
\end{gathered}
$$


Hence, the inner feedback loop of the electrical domain describes a first-order lag with a constant parameter $2 / \tau_{p}$. To obtain an expression for the mechanical power $p_{\text {mech }}$ and by this for the feedback of the mechanical on the electrical domain, the force $F_{e l}=A \cdot \sigma_{e l}$ using equations (1) and (12) as well as the velocity $\dot{\Delta} z=z_{0} \cdot \dot{\varepsilon}_{z}$ has to be considered

$$
\begin{aligned}
p_{\text {mech }} & =\frac{2}{V} \cdot U_{c, \text { diel }} \cdot \frac{A_{0}}{1-\varepsilon_{z}} \cdot z_{0} \cdot \dot{\varepsilon}_{z} \\
& =2 \cdot U_{c, \text { diel }} \cdot \frac{\dot{\varepsilon}_{z}}{1-\varepsilon_{z}}, \text { with } V=A_{0} \cdot z_{0}
\end{aligned}
$$

Thus, this feedback only depends on $U_{c, \text { diel }}$ and the strain state. As can be seen by this equation, the power $p_{\text {mech }}$ occurs only for $\dot{\varepsilon}_{z} \neq 0$ and $U_{c, \text { diel }} \neq 0$. It describes an actuation in case of $\dot{\varepsilon}_{z}>0$ and a harvesting of mechanical energy for $\dot{\varepsilon}_{z}<0$ (generator mode). Furthermore, typical materials used for DE applications have a high specific resistance $\rho_{p}$. Due to the resulting high time constant $\tau_{p}, \bar{p}^{\prime}$ and $p_{\text {mech }}$ are the dominant parts in equation (15). For simplified control designs, it might be sufficient to neglect $p_{R p}$, while here it is taken into account to obtain a general design. Finally, this results in the overall nonlinear action diagram of the DE stack-actuator in Figure 6 comprising the coupled (yellow box) mechanical (green box) and electrical domain (red box).

Furthermore, under consideration of the action diagram, a state vector $x_{D E}$ can be defined consisting of the strain $\varepsilon_{z}$, its velocity $\dot{\varepsilon}_{z}$, the strain $\varepsilon_{k 1}$, and the energy $U_{c, \text { diel }}$

$$
x_{D E}^{T}=\left[\begin{array}{llll}
\varepsilon_{z} & \dot{\varepsilon}_{z} & \varepsilon_{k 1} & U_{c, \text { diel }}
\end{array}\right]
$$

Here, $U_{c, \text { diel }}$ is chosen instead of the voltage $v_{p}$ due to the linear relation in equation (1).

Defining the input

$$
u=\bar{p}^{\prime}
$$

this yields the nonlinear differential equations

$$
\begin{gathered}
\dot{\mathbf{x}}_{D E}=\left[\begin{array}{c}
\dot{\varepsilon}_{z} \\
\ddot{\varepsilon}_{z} \\
\dot{\varepsilon}_{k 1} \\
\dot{U}_{c, \text { diel }}
\end{array}\right]=\underbrace{\left[\begin{array}{l}
f_{1, D E}\left(\mathbf{x}_{D E}\right) \\
f_{2, D E}\left(\mathbf{x}_{D E}\right) \\
f_{3, D E}\left(\mathbf{x}_{D E}\right) \\
f_{4, D E}\left(\mathbf{x}_{D E}\right)
\end{array}\right]}_{\mathrm{f}_{D E}\left(\mathbf{x}_{D E}\right)}+\mathbf{B}_{D E} \cdot u \\
=\left[\begin{array}{c}
\frac{V}{\dot{\varepsilon}_{z}} \cdot \frac{\sigma_{a c t}-\sigma_{\text {load }}}{1-\varepsilon_{z}} \\
m_{a c c} \cdot z_{0}^{2} \\
\dot{\varepsilon}_{z}-\frac{k_{1}}{\eta_{1}} \cdot \varepsilon_{k 1} \\
-2 \cdot U_{c, \text { diel }} \cdot\left(\frac{\dot{\varepsilon}_{z}}{1-\varepsilon_{z}}+\frac{1}{\tau_{p}}\right)
\end{array}\right]+\left[\begin{array}{l}
0 \\
0 \\
0 \\
1
\end{array}\right] \cdot \bar{p}^{\prime}, \\
\text { with } \sigma_{a c t}=\frac{2}{V} \cdot U_{c, \text { diel }}-\frac{Y}{3} \cdot\left(\frac{1}{1-\varepsilon_{z}}-\left(1-\varepsilon_{z}\right)^{2}\right) \\
-\eta_{E} \cdot \dot{\varepsilon}_{z}-k_{1} \cdot \varepsilon_{k 1}
\end{gathered}
$$

\section{Force control design}

As explained above, the input $\bar{p}^{\prime}$ of the control plant can vary between three discrete values only, that is, the DE transducer is charged $\left(\bar{p}^{\prime}=+\bar{p}_{\max }\right)$, held in its current state $\left(\bar{p}^{\prime}=0\right)$, or discharged $\left(\bar{p}^{\prime}=-\bar{p}_{\max }\right)$, see equation (4). Due to this property, a variable structure control design is advantageous for the intended force control that has to decide which of the input states is required to reach the set-point $F_{a c t}^{*}$ of the desired force. The strongly nonlinear behavior of the control plant in Figure 6 requires a particular control strategy to ensure robustness and sufficient accuracy especially in combination with high dynamics. Thus, a sliding mode force controller (SMC) as described by DeCarlo et al. (1988) is chosen, since it fulfills these specifications and it is predestined for the properties of the considered control plant. The idea of this approach is to define a switching surface in the state space of the system on which the system state is led toward a desired steady-state setpoint value.

\section{Design of a sliding mode force control}

One of the key aspects of a SMC is the definition of a switching function $S(\mathbf{x})$. In case of a system of $n$th order with $p=1$ input usually a linear switching function (DeCarlo et al., 1988) is chosen by

$$
S(\Delta \mathbf{x})=\mathbf{c}^{T} \cdot \Delta \mathbf{x}, \text { with } \mathbf{c}^{T}=\left[\begin{array}{lll}
c_{1} & \cdots & c_{n}
\end{array}\right]
$$

On the switching surface, the trajectory of the system is moved toward the origin containing the set-point values during the so-called sliding mode by ensuring $S(\Delta \mathbf{x})=0$. To conduct the system toward any arbitrary point in the state space, the state variables are transformed to $\Delta \mathbf{x}=\hat{\mathbf{x}}-\mathbf{x}^{*}$ as depicted in Figure 7, where $\mathbf{x}^{*}$ represents the set-point state vector and $\hat{\mathbf{x}}$ contains all current state variables estimated with an EKF based on the measured quantities as explained later on. Furthermore, the utilized control law and an adaption approach under consideration of the driving power converter and limiting conditions are schematically depicted in Figure 7 on the right-hand side and explained in detail in the following section.

Under consideration of the model summarized in the previous section, set-point values for all four state variables $\varepsilon_{z}^{*}, \dot{\varepsilon}_{z}^{*}, \varepsilon_{k 1}^{*}$, and $U_{c, \text { diel }}^{*}$, respectively, have to be defined. While the velocity $\dot{\varepsilon}_{z}^{*}=0$ and the strain of the spring in the Maxwell element $\varepsilon_{k 1}^{*}=0$ are zero at steady state, the other two state variables are not equal to zero. During steady state, the actuator force is in equilibrium with the external load 


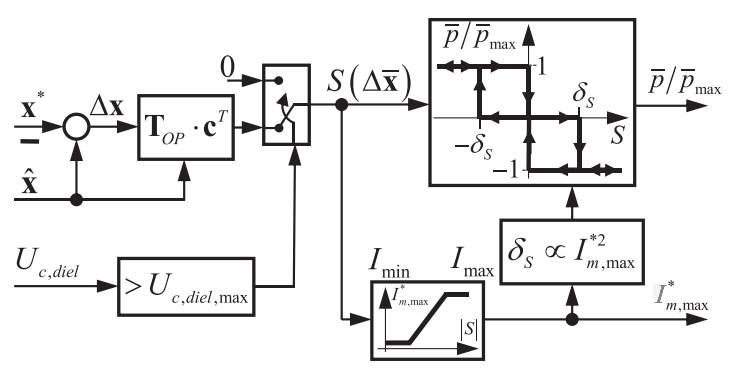

Figure 7. Structure of the SMC under consideration of the bidirectional flyback-converter as control element.

$$
\begin{aligned}
& F_{\text {act }}^{*}=\lim _{t \rightarrow \infty} A \cdot \sigma_{\text {act }}(t) \stackrel{!}{=} A \cdot \sigma_{\text {load }} \\
& \text { with } \lim _{t \rightarrow \infty} A \cdot \sigma_{\text {act }}(t)=A_{e} \cdot \sigma_{\text {el }}-A \cdot \sigma_{\text {elast }}
\end{aligned}
$$
by

With equation (10), a set-point energy $U_{c, \text { diel }}^{*}$ is given

$$
U_{c, \text { diel }}^{*}=\frac{V}{2} \cdot\left(\frac{F_{\text {act }}^{*}}{A}+\sigma_{\text {elast }}\right)=\frac{z}{2} \cdot\left(F_{\text {act }}^{*}+A \cdot \sigma_{\text {elast }}\right)
$$

Beside the set-point force $F_{a c t}^{*}$ this energy is influenced by the elastic material tensions. Thus, they have to be compensated in order to obtain a certain force under deformation, compare Figure 2(b). The required energy has to be adapted continuously depending on the actual strain and the resulting elastic tension according to equation (6). For this purpose, here the estimated strain $\hat{\varepsilon}_{z}$ obtained from the EKF explained afterward is used.

The intention of the proposed force control is to adjust the set-point force independently of the actuation strain. Nevertheless, depending on the kind of load, for example, a spring, the DE stack-actuator will deform when a certain force is adjusted by the control. Then, according to equation (23), $U_{c, \text { diel }}^{*}$ will change during transient operation. Thus, as a starting point either the current strain or for example the half of the maximum strain achievable with the desired force $F_{a c t}^{*}$ can be chosen. Considering for example the dasheddotted spring load line in Figure 3(b), this statistical mean strain might result in a faster convergence to the steady-state energy improving the dynamics of the control.

Finally, the definition of a set-point strain contradicts the mentioned intention of the control of adjusting a force independently of the strain. To solve this issue, subsequently the strain is interpreted as a disturbance. This has the big advantage that the state variables for the force control design can be reduced by the strain so that no set-point strain is required. Instead, its influence is compensated by the adaptation of the setpoint energy according to equation (23). The resulting reduced system of third order is given by

$$
\dot{\mathbf{x}}=\left[\begin{array}{c}
\ddot{\varepsilon}_{z} \\
\dot{\varepsilon}_{M W} \\
\dot{U}_{c, \text { diel }}
\end{array}\right]=\underbrace{\left[\begin{array}{c}
f_{2, D E}\left(\mathbf{x}_{D E}\right) \\
f_{3, D E}\left(\mathbf{x}_{D E}\right) \\
f_{4, D E}\left(\mathbf{x}_{D E}\right)
\end{array}\right]}_{\mathrm{f}\left(\mathbf{x}_{D E}\right)}+\underbrace{\left[\begin{array}{c}
0 \\
0 \\
1
\end{array}\right]}_{B} \cdot u
$$

Under consideration of equation (23), the set-point state vector $\mathbf{x}^{*}$ is given in this case by

$$
\mathbf{x}^{*}=\left[\begin{array}{c}
\dot{\varepsilon}_{z}^{*} \\
\dot{\varepsilon}_{k 1}^{*} \\
U_{c, \text { diel }}^{*}
\end{array}\right]=\left[\begin{array}{c}
0 \\
0 \\
f\left(F_{a c t}^{*}, \hat{\varepsilon}_{z}\right)
\end{array}\right]
$$

The transformed state vector $\Delta \mathbf{x}$ for the calculation of the switching function in equation (21) is calculated with the current state vector $\mathbf{x}$ and the set-point vector $\mathbf{x}^{*}$

$$
\begin{aligned}
\Delta \mathbf{x} & =\mathbf{x}-\mathbf{x}^{*} \\
& =\left[\begin{array}{c}
\dot{\varepsilon}_{z} \\
\dot{\varepsilon}_{k 1} \\
U_{c, \text { diel }}
\end{array}\right]-\left[\begin{array}{c}
0 \\
0 \\
U_{c, \text { diel }}^{*}
\end{array}\right]=\left[\begin{array}{c}
\dot{\varepsilon}_{z} \\
\dot{\varepsilon}_{k 1} \\
\Delta U_{c, \text { diel }}
\end{array}\right]
\end{aligned}
$$

A comparable simple approach to determine the coefficients in $\mathbf{c}^{T}$ is based on the controllable canonical form as proposed by DeCarlo et al. (1988). In this form, the input $u$ affects one state variable only, while the others are independent of the input. To transform the system into this form, first of all the system has to be linearized described by the matrix $\mathbf{A}_{O P}$ of the reduced system in equation (24) and calculated by the Jacobian matrix of $\mathbf{f}(\mathbf{x})$ with respect to $\mathbf{x}$ for the current operating point (OP)

$$
\begin{aligned}
& \mathbf{A}_{O P}=\frac{\partial \mathbf{f}(\mathbf{x})}{\partial \mathbf{x}} \\
& =\left[\begin{array}{ccc}
-\gamma_{1} \cdot \frac{\eta_{e}}{1-\hat{\varepsilon}_{z}} & -\gamma_{1} \cdot \frac{k_{1}}{1-\hat{\varepsilon}_{z}} & \gamma_{1} \cdot \frac{2 / V}{1-\hat{\varepsilon}_{z}} \\
1 & -\frac{k_{1}}{\eta_{1}} & 0 \\
-\frac{2 \cdot U_{c, \text { diel }}}{1-\hat{\varepsilon}_{z}} & 0 & -\frac{2 \cdot \hat{\varepsilon}_{z}}{1-\hat{\varepsilon}_{z}}-\frac{2}{\tau_{p}}
\end{array}\right] \text { OP } \\
& \text { with } \gamma_{1}=\frac{V}{m_{\text {acc }} \cdot z_{0}^{2}}
\end{aligned}
$$

The controllability is given because the determinant of the matrix $\Gamma_{c}$ is not equal to zero for any arbitrary operating point calculated by (Kalman, 1960)

$$
\begin{aligned}
\operatorname{det}\left(\boldsymbol{\Gamma}_{c}\right) & =\operatorname{det}\left(\left[\begin{array}{lll}
\mathbf{B} & \mathbf{A}_{O P} \cdot \mathbf{B} & \mathbf{A}_{O P}^{2} \cdot \mathbf{B}
\end{array}\right]\right) \\
& =\frac{4 \cdot \gamma_{1}^{2}}{V^{2} \cdot\left(1-\hat{\varepsilon}_{z}\right)^{2}} \neq 0, \text { with } \hat{\varepsilon}_{z}<1
\end{aligned}
$$

Using the last row $\gamma_{c 3}$ of $\boldsymbol{\Gamma}_{c}$, the transformation matrix $\mathbf{T}_{O P}$ for a certain operating point is given by

$$
\begin{aligned}
& \mathbf{T}_{O P}=\left[\begin{array}{c}
\gamma_{c 3} \\
\gamma_{c 3} \cdot \mathbf{A}_{O P} \\
\gamma_{c 3} \cdot \mathbf{A}_{O P}^{2}
\end{array}\right] \\
& =-\frac{V}{2} \cdot\left[\begin{array}{ccc}
0 & -\frac{1-\hat{\varepsilon}_{z}}{\gamma_{1}} & 0 \\
-\frac{1-\hat{\varepsilon}_{z}}{\gamma_{1}} & \frac{k_{1}}{\eta_{1}} \cdot \frac{1-\hat{\varepsilon}_{z}}{\gamma_{1}} & 0 \\
\frac{k_{1} \cdot\left(1-\hat{\varepsilon}_{z}\right)}{\gamma_{1} \cdot \eta_{1}}+\eta_{e} & \frac{k_{1}}{\eta_{1}}\left(\eta_{1}-\frac{k_{1} \cdot\left(1-\hat{\varepsilon}_{z}\right)}{\gamma_{1} \cdot \eta_{1}}\right) & -\frac{2}{V}
\end{array}\right]
\end{aligned}
$$


With this transformation matrix, the state vector $\Delta \mathbf{x}$ defined in equation (26) can be transformed into the controllable canonical form by

$$
\Delta \overline{\mathbf{x}}=\mathbf{T}_{O P} \cdot \Delta \mathbf{x}=\mathbf{T}_{O P} \cdot\left(\mathbf{x}-\mathbf{x}^{*}\right)
$$

while with the system matrix $\overline{\mathbf{A}}_{O P}=\mathbf{T}_{O P} \cdot \mathbf{A}_{O P} \cdot \mathbf{T}_{O P}^{-1}$ and the input matrix $\overline{\mathbf{B}}=T_{O P} \cdot \mathbf{B}=\mathbf{B}$, the transformed dynamics result in

$$
\Delta \dot{\overline{\mathbf{x}}}=\overline{\mathbf{A}}_{O P} \cdot \Delta \overline{\mathbf{x}}+\mathbf{B} \cdot u
$$

In order to lead the system along the switching surface by $S(\Delta \overline{\mathbf{x}})=0$, the so-called equivalent input $u=u_{e q}$ (DeCarlo et al., 1988) in equation (32) can be derived. To remain on the switching surface during the sliding mode the derivative of the switching function $\dot{S}(\Delta \overline{\mathbf{x}})=0$ that has to be zero. This results in

$$
u_{e q}=-\left[\mathbf{c}^{T} \cdot \mathbf{B}\right]^{-1} \cdot \mathbf{c}^{T} \cdot \overline{\mathbf{A}}_{O P} \cdot \Delta \overline{\mathbf{x}}
$$

Using this input, the dynamics of the system during the sliding mode are reduced by the number $p$ of input quantities (here $p=1$ ). Furthermore, the dynamics only depends on the chosen parameters of $\mathbf{c}^{T}$ according to equation (21) and is independent of any parameters of the control plant

$$
\begin{aligned}
\Delta \dot{\overline{\mathbf{x}}} & =\left[\mathbf{I}-\mathbf{B} \cdot\left[\mathbf{c}^{T} \cdot \mathbf{B}\right]^{-1} \cdot \mathbf{c}^{T}\right] \cdot \overline{\mathbf{A}}_{O P} \cdot \Delta \overline{\mathbf{x}} \\
& =\left[\begin{array}{ccc}
0 & 0 & 0 \\
0 & 0 & 1 \\
0 & -\frac{c_{1}}{c_{3}} & -\frac{c_{2}}{c_{3}}
\end{array}\right] \cdot \Delta \overline{\mathbf{x}}=\left[\begin{array}{cc}
0 & 0^{T} \\
0 & \tilde{\mathbf{A}}_{1}
\end{array}\right] \cdot \Delta \overline{\mathbf{x}}
\end{aligned}
$$

By applying for example a pole placement for the reduced system described by matrix $\tilde{\mathbf{A}}_{1}$, the coefficients of the switching function in equation (21) can be determined by comparing the characteristic equation to an arbitrary second-order polynomial defined in the $s$ domain with cutoff frequency $\omega_{c}$ and damping ratio $D$

$$
\begin{aligned}
& \operatorname{det}\left(s \cdot \mathbf{I}-\tilde{\mathbf{A}}_{1}\right)=s^{2}+\frac{c_{2}}{c_{3}} \cdot s+\frac{c_{1}}{c_{3}} \\
& =s^{2}+2 \cdot D \cdot \omega_{c} \cdot s+\omega_{c}^{2} \\
& \text { with } c_{3}=1 \quad \Rightarrow c_{1}=\omega_{c}^{2}, c_{2}=2 \cdot D \cdot \omega_{c}
\end{aligned}
$$

Due to the reduced order during the sliding mode, one state variable, for example $\Delta U_{c, \text { diel }}=U_{c \text {, diel }}-U_{c, \text { diel }}^{*}$, can be expressed by the others under consideration of equations (25) and (29)

$$
\begin{aligned}
& S(\Delta \overline{\mathbf{x}})=\mathbf{c}^{T} \cdot \mathbf{T} \cdot\left(\mathbf{x}-\mathbf{x}^{*}\right)=0 \\
& \text { with } \mathbf{c}^{T} \cdot \mathbf{T}=\left[\begin{array}{lll}
\bar{c}_{1} & \bar{c}_{2} & \bar{c}_{3}
\end{array}\right] \\
& \bar{c}_{1}=\frac{V}{2} \cdot\left[\frac{1-\hat{\varepsilon}_{z}}{\gamma_{1}} \cdot\left(2 D \omega_{c}-\frac{k_{1}}{\eta_{1}}\right)-\eta_{e}\right] \\
& \bar{c}_{2}=\frac{V}{2} \cdot\left[\frac{1-\hat{\varepsilon}_{z}}{\gamma_{1}} \cdot\left(\omega_{c}^{2}-\frac{k_{1}}{\eta_{1}} \cdot\left(2 D \omega_{c}-\frac{k_{1}}{\eta_{1}}\right)\right)-k_{1}\right] \\
& \bar{c}_{3}=1 \\
& \Rightarrow \Delta U_{c, \text { diel }}=-\bar{c}_{1} \cdot \dot{\varepsilon}_{z}-\bar{c}_{2} \cdot \varepsilon_{k 1}
\end{aligned}
$$

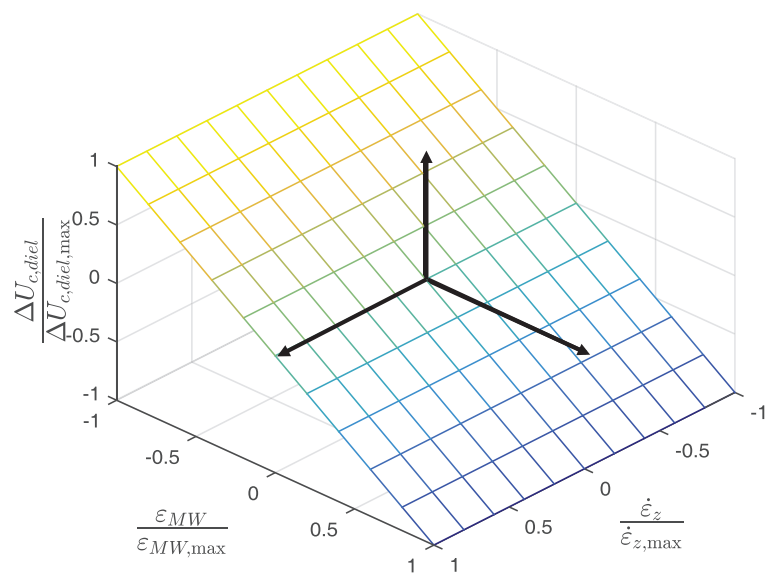

Figure 8. Exemplary illustrated sliding surface $S(\Delta \overline{\mathbf{x}})=0$ for the considered system.

This results in the exemplary switching surface depicted in Figure 8, whose slope can be adjusted by the chosen cutoff frequency $\omega_{c}$ in equation (34) defining the coefficients of the switching function. Here, the material parameters of the silicone-based DE stackactuator, listed in the following validation section, have been used.

While the behavior during the sliding mode is already determined, the controller function has to be parametrized ensuring that the sliding mode is reached in a first step from any arbitrary starting point $\Delta \overline{\mathbf{x}}\left(t_{0}\right)$ during the so-called reaching phase. For this purpose, the Lyapunov stability for a single-input single-output (SISO)-system is proven

$$
\dot{V}(\Delta \overline{\mathbf{x}})=S(\Delta \overline{\mathbf{x}}) \cdot \dot{S}(\Delta \overline{\mathbf{x}}) \stackrel{!}{<} 0
$$

Thus, if the switching function $S(\Delta \overline{\mathbf{x}})$ with the current state vector $\Delta \overline{\mathbf{x}}$ for example is larger than zero, its time derivative has to be negative in order to reach the sliding mode and vice versa.

Due to the characteristic properties of the utilized flyback-converter, it is possible to charge or discharge the DE transducer with an almost constant power $\bar{p}^{\prime}$. In a first step, this behavior is taken into account by the two-point controller in equation (37)

$$
u(\Delta \overline{\mathbf{x}})=\operatorname{sgn}(S(\Delta \overline{\mathbf{x}})) \cdot \varrho
$$

Here, $\varrho$ either equals the positive or negative feeding power, respectively, and has to be chosen to fulfill condition (equation (36)). For this purpose, the time derivative of the switching function is determined

$$
\begin{aligned}
\dot{S}(\Delta \overline{\mathbf{x}}) & =\mathbf{c}^{T} \cdot \mathbf{T} \cdot\left(\mathbf{A}_{O P} \cdot \Delta \mathbf{x}+\mathbf{B} \cdot u(\Delta \overline{\mathbf{x}})\right) \\
& =\zeta_{1} \cdot \dot{\varepsilon}_{z}+\zeta_{2} \cdot \varepsilon_{k 1}+\zeta_{3} \cdot U_{c, \text { diel }}+u(\Delta \overline{\mathbf{x}})
\end{aligned}
$$

The coefficients $\zeta_{i}$ depend on material and design parameters of the stack-transducer as well as on the 
damping ratio $D$ and cutoff frequency $\omega_{c}$ according to equation (34). Hence, these two design parameters of the controller can be chosen in such a way that two coefficients in equation (38) vanish. By choosing $\zeta_{1}=0$ and $\zeta_{2}=0$, equation (38) solely depends on the energy $\Delta U_{c, \text { diel }}=U_{c, \text { diel }}-U_{c, \text { diel }}^{*}$ and he input $u(\Delta \overline{\mathbf{x}})$. For the operating point $\mathbf{x}_{O P}=\mathbf{0}$, the cutoff frequency $\omega_{c}$ and damping ratio $D$ in this case are given by

$$
\begin{gathered}
\omega_{c}=\frac{1}{\eta_{1}} \cdot \sqrt{\gamma_{1} \cdot k_{1} \cdot \eta_{1} \cdot\left(\eta_{1}+\eta_{E}\right)} \\
D=\frac{1}{2} \cdot \sqrt{\frac{\left(k_{1}+\gamma_{1} \cdot \eta_{1} \cdot \eta_{E}\right)^{2}}{\gamma_{1} \cdot k_{1} \cdot \eta_{1} \cdot\left(\eta_{1}+\eta_{E}\right)}}
\end{gathered}
$$

Substituting these into equation (38), the time derivative of the switching function simplifies to

$$
\dot{S}(\Delta \overline{\mathbf{x}})=-\frac{2}{\tau_{p}} \cdot \Delta U_{c, \text { diel }}+u(\Delta \overline{\mathbf{x}})
$$

By carrying out the required case distinction for the condition in equation (36) with equations (37) and (40), it can be found out that $\varrho=-\bar{p}_{\max }$ fulfills equation (36) if the maximum feeding power $\bar{p}_{\max }$ is large enough to compensate the dielectric loss in the utilized polymer, compare equation (16). However, for a well-designed smart actuator system, this condition should be fulfilled

$$
\bar{p}_{\max }>\frac{2}{\tau_{p}} \cdot\left|\Delta U_{c, \text { diel }}\right|
$$

It can be numerically proven that equation (41) is fulfilled for any arbitrary operating point $\mathbf{x}_{O P} \neq \mathbf{0}$.

\section{Adaptation of the force control}

Under consideration of the defined switching function in equation (35)

$$
S(\Delta \overline{\mathbf{x}})=\bar{c}_{1} \cdot \dot{\varepsilon}_{z}+\bar{c}_{2} \cdot \varepsilon_{k 1}+\Delta U_{c, \text { diel }}
$$

an adaption of the force control can be carried out to reduce the switching frequency and to take into account limitations of the control plant.

The latter have to be considered in order to not exceed the dielectric breakdown strength of the utilized elastomer material. Under consideration of the utilized flyback-converter and the maximum allowable voltage $v_{p, \max }$ to prevent a breakdown, the voltage $v_{p}$ across the polymer capacitance $C_{p}$ has to be adjusted in the range $0 \leq v_{p} \leq v_{p, \max }$ corresponding to the energy range $0 \leq U_{c, \text { diel }} \leq U_{c, \text { diel, } \text { max }}$ according to equation (16b). If the energy exceeds this range, the control is switched off by the extension also depicted in Figure 7

$$
S(\Delta \overline{\mathbf{x}})=\left\{\begin{array}{l}
\mathbf{c}^{T} \cdot \Delta \overline{\mathbf{x}}, \quad U_{c, \text { diel }} \leq U_{c, \text { diel, }, \text { max }} \\
0, \quad \text { else }
\end{array}\right.
$$

To avoid a permanent switching during the sliding mode and steady state, the two-point controller can be extended by a hysteresis in order to reduce switching losses. Furthermore, the third state of the flyback-converter, the off-state, can additionally be used to establish a three-point controller with hysteresis as depicted in Figure 7. The hysteresis threshold $\delta_{S}$ results in two switching functions in parallel above and below the switching surface $S(\Delta \overline{\mathbf{x}})=0$ in Figure 8 with distance $\delta_{S}$, that is, the trajectory of the system does not move exactly on the switching surface, but within the certain space defined by $|S(\Delta \overline{\mathbf{x}})|<\delta_{S}$. However, during steady state, this threshold results in a control deviation so that it should be chosen carefully. Within one switching period of the utilized flyback-converter, the energy increment $\Delta U_{\max }$ is transferred and charges or discharges the DE actuator. In case of the steady state with $\dot{\varepsilon}_{z}=0$ and $\varepsilon_{k 1}=0$, the switching function (equation (42)) only depends on the energy deviation $\Delta U_{c \text {, diel }}$. With this, the threshold $\delta_{S}$ can be determined by inserting $\Delta U_{c, \text { diel }}=n_{\delta s} \cdot \Delta U_{\max }$ into equation (42). Thus, the threshold can be chosen as an integer multiple of the transferred energy increment $\Delta U_{\max }$ in equation (3)

$$
\delta_{S}=n_{\delta s} \cdot \Delta U_{\max }=n_{\delta s} \cdot \frac{1}{2} \cdot L_{m} \cdot I_{m, \max }^{* 2}
$$

Here, $n_{\delta s}$ should be as small as possible to obtain good control accuracy, and in contrast as large as necessary to sufficiently reduce the switching frequency.

These two modifications result in the trajectories of the switching functions schematically depicted in Figure 9 for a sectional view on the $\varepsilon_{k 1}-\Delta U_{c \text {, diel }}$ plane.

As can be seen in equation (44), the hysteresis threshold $\delta_{S}$ that determines the control accuracy during steady state depends on the peak value of the magnetizing current $I_{m, \max }^{*}$. However, with the control of the bidirectional flyback-converter presented in Figure 4, it is possible to adjust this peak value, see Figure 5. In terms of the force control, a high value of the current should be chosen for high dynamics during transient operation while a small quantity during steady state improves the accuracy of the control.

During the reaching phase, where the trajectory is led toward the switching surface, the error amount with respect to the switching function $|S(\Delta \overline{\mathbf{x}})|$ is comparable high, while during the sliding mode and steady state it is comparable low. Thus, by defining an upper and lower threshold $S_{\min }$ and $S_{\max }$, respectively, the peak value of the current $I_{m, \text { max }}^{*}$ can be adjusted depending on $|S(\Delta \overline{\mathbf{x}})|$

$$
I_{m, \max }^{*}=\left\{\begin{array}{l}
I_{\max },|S(\Delta \overline{\mathbf{x}})|>S_{\max } \\
I_{\max }-I_{\min } \cdot|S(\Delta \overline{\mathbf{x}})|, \text { else } \\
S_{\operatorname{mix}}-S_{\min }(\Delta \overline{\mathbf{x}}) \mid<S_{\min }
\end{array}\right.
$$

This current adjustment is finally used as set-point for the inner current control of the flyback-converter as 
well as for the calculation of $\delta_{S}$ in equation (44) as already depicted in Figure 7.

\section{Estimation of the transducer states}

To implement the developed force control, the state variables of the DE stack-transducer must be known. However, as measured quantities solely the terminal voltage $v_{D E, m}$ and the compression $\Delta z_{m}$ of the stack are available. Hence, a state estimator is used that estimates the energy $\hat{U}_{c, \text { diel }}$ in a first step. In a second step, the mechanical states of the DE actuator are estimated. This two-step procedure is used instead of an estimator for the overall model in equation (20) since the observability of this system is not given for any arbitrary operating point.

The energy $\hat{U}_{c \text {, diel }}$ stored in the strain-dependent polymer capacitance $C_{p}$ depends on the charge $\hat{q}_{p}$ or voltage $\hat{v}_{p}$, respectively

$$
\hat{U}_{c, \text { diel }}=\frac{\hat{q}_{p}^{2}}{2 \cdot C_{p}\left(\hat{\varepsilon}_{z}\right)} \text {, with } \hat{q}_{p}=\frac{C_{p, 0}}{\left(1-\hat{\varepsilon}_{z}\right)^{2}} \cdot \hat{v}_{p}
$$

Considering an equi-biaxial in-plane deformation of the stack-transducer, the capacitance $C_{p}=C_{p, 0} /$ $\left(1-\varepsilon_{z}\right)^{-2}$ depends on its undeformed value $C_{p, 0}$ and the strain $\varepsilon_{z}$. The electrode resistance $R_{e}$ marginally varies within the possible deformation range so that it is assumed to be constant. With the equivalent circuit diagram in Figure 1 (red box), the change in charge is described by the differential equation

$$
\dot{\hat{q}}_{p}=\frac{v_{D E, m}}{R_{e}}-\left(\frac{1}{\tau_{p}}+\frac{\left(1-\hat{\varepsilon}_{z}\right)^{2}}{R_{e} \cdot C_{p, 0}}\right) \cdot \hat{q}_{p}
$$

Here, the strain $\hat{\varepsilon}_{z}=\Delta z_{m} / z_{0}=\left(z-z_{0}\right) / z_{0}$ is calculated using the measured compression and initial transducer height $\Delta z_{m}$ and $z_{0}$, respectively, as depicted in the action diagram in Figure 10.

The estimated energy $\hat{U}_{c \text {, diel }}$ is then used as input for an EKF implemented according to Welch and Bishop (2001) that observes the mechanical states of the DE stack-actuator as well as the load tension $\hat{\sigma}_{\text {load }}$ as disturbance (Hoffstadt and Maas, 2017). Although an estimate of the load is not required for the control, it has to be considered when designing the EKF to avoid larger deviations of the other state quantities. Beside the applied energy $U_{c, \text { diel }}$, this disturbance mainly influences the behavior of the DE actuator. Under consideration of the corresponding differential equation (20) and assuming the disturbance to be constant during one sampling time $T=1 / f_{D S P}$ the estimated state vector $\hat{\mathbf{x}}_{E K F}$ is given by

$$
\hat{\mathbf{x}}_{E K F}=\left[\begin{array}{llll}
\hat{\varepsilon}_{z} & \dot{\hat{\varepsilon}}_{z} & \hat{\varepsilon}_{M W} & \hat{\sigma}_{\text {load }}
\end{array}\right]^{T}
$$

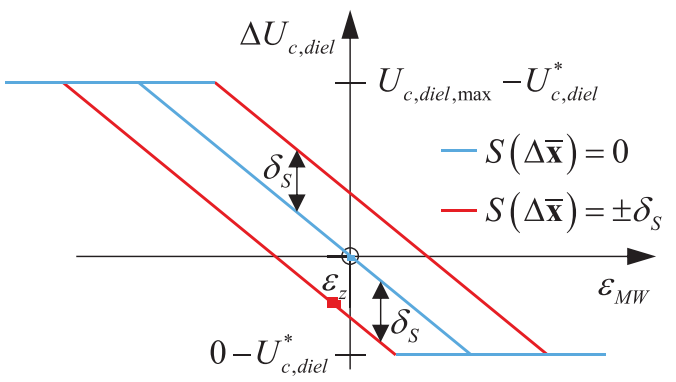

Figure 9. Implemented switching function for the three-point controller with hysteresis considering control output limitations.

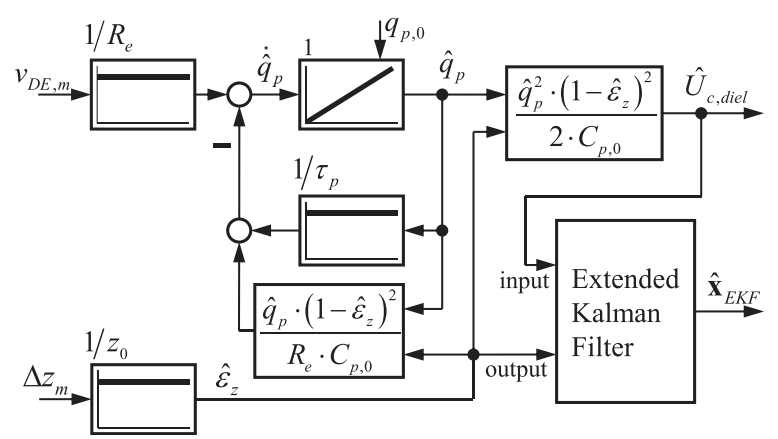

Figure 10. Based on the measured terminal voltage $v_{D E, m}$ and deformation $\Delta z_{m}$, the mechanical transducer states $\hat{\mathbf{x}}$ and a disturbance $\hat{\sigma}_{\text {load }}$ are estimated.

Finally, the state vector $\hat{\mathbf{x}}=\left[\begin{array}{lll}\dot{\hat{\varepsilon}}_{z} & \hat{\varepsilon}_{M W} & \hat{U}_{c, \text { diel }}\end{array}\right]^{T}$ required for the force control in Figure 7 comprises the estimated velocity $\dot{\hat{\varepsilon}}_{z}$, the strain $\hat{\varepsilon}_{M W}$, and energy $\hat{U}_{c, \text { diel }}$.

\section{Experimental validation}

For the experimental validation of the proposed sliding mode force control, the test-rig depicted in Figure 11 as well as a prototype of the bidirectional flybackconverter and a DE stack-actuator is used. The parameters of the silicone-based stack-actuator with two mechanical interfaces on its top and bottom surface to transmit the actuator force are listed in Table 1. These parameters result in a cutoff frequency of $\omega_{c} \approx 3060 \mathrm{~s}^{-1}$ and damping ratio of $D \approx 1.08$ when applying equations (39a) and (39b), respectively. With the linear motor of the test-rig, the stack-actuator can be deformed, while the measurement of the force is solely recorded for the validation but is not required for the force control itself.

Before the validation of the proposed force control is carried out, the open-loop behavior of a DE stackactuator is investigated. In the bottom plot of Figure 12 , the blocking force (solid lines) as well as the no-load strain (dashed lines) are compared for a charging 


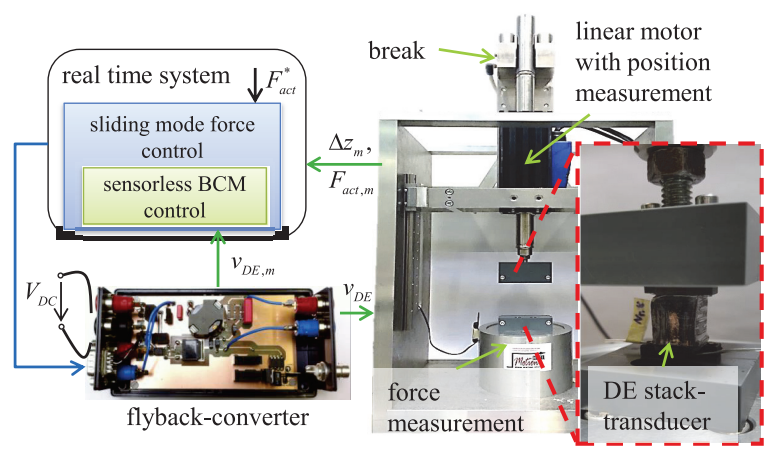

Figure I I. Overview of the test setup utilized for the validation comprising a specifically developed prototype of the bidirectional flyback-converter, a silicone-based DE stacktransducer, and a test-rig with force measurement and electrical linear motor.

operation with $I_{m, \max }^{*}=8 \mathrm{~A}$ (blue) and $I_{m, \max }^{*}=4 \mathrm{~A}$ (red), respectively. The blocking force represents the actuator force $F_{a c t}$ obtained when the deformation of the actuator is blocked at $\varepsilon_{z}=0$, while the no-load strain characterizes the free deformation with $F_{\text {act }}=0$. As can be seen, the blocking force follows instantaneously the voltage signal, while in case of the strain a very short delay caused by the viscosity of the DE material can be observed.

For a first experimental investigation of the proposed closed-loop control, one interface of the DE actuator is attached to the force measurement system of the test-rig and the second interface to the slider of the linear motor. Fixing the slider of the linear motor by an internal brake, the stack-actuator cannot deform $\left(\varepsilon_{z}=0\right)$ even if a voltage is applied. With this configuration, the blocking force can be characterized. Due to the constant deformation $\left(\varepsilon_{z}=0\right)$, the viscoelastic material properties do not influence these investigations. Furthermore, this setup is used to compare the two-point controller in equation (37) with the proposed three-point controller with hysteresis, as shown in Figure 7. The obtained measurement results are depicted in Figure 13. By reducing the peak current from $I_{m, \max }^{*}=8 \mathrm{~A}$ (blue) to $I_{m, \max }^{*}=4 \mathrm{~A}$ (green) with the two-point controller $\left(\delta_{S}=0\right)$, it takes longer to adjust the set-point force $F_{a c t}^{*}$ due to the reduced feeding power $\bar{p}$ according to equations (3) and (4). However, as mentioned in the previous section during steady state, the control accuracy is improved resulting in a reduction of the standard deviation of the force, for example, in the interval between 55 and $80 \mathrm{~ms}$ of about $25 \%$ from $41 \mathrm{mN}\left(I_{m, \max }^{*}=8 \mathrm{~A}\right)$ to $31 \mathrm{mN}$ $\left(I_{m, \max }^{*}=4 \mathrm{~A}\right)$. Instead of the energy $U_{c, \text { diel }}$ here the corresponding voltage $v_{D E}$ is shown in the third subplot that of course depends on the set-point force. Due to blocking-force scenario where a deformation is prevented, the set-point energy $U_{c, \text { diel }}^{*}$ in equation (23)
Table I. Parameters of the utilized silicone stack-transducer.

\begin{tabular}{llll}
\hline$Y(\mathrm{MPa})$ & $\eta_{\mathrm{e}}(\mathrm{kPa} \mathrm{s})$ & $k_{1}(\mathrm{MPa})$ & $\eta_{1}(\mathrm{kPa} \mathrm{s})$ \\
1.08 & 6 & 5 & 1 \\
\hline$A_{e}\left(\mathrm{~mm}^{2}\right)$ & $A_{0}\left(\mathrm{~mm}^{2}\right)$ & $d_{0}(\mu \mathrm{m})$ & $\mathrm{N}$ \\
64 & 100 & 50 & 192 \\
\hline$\varepsilon_{r}$ & $C_{p, 0}(\mathrm{nF})$ & $R_{\mathrm{e}}(\mathrm{k} \Omega)$ & $\tau_{p}(\mathrm{~s})$ \\
2.8 & 6.09 & 30 & 2670 \\
\hline
\end{tabular}
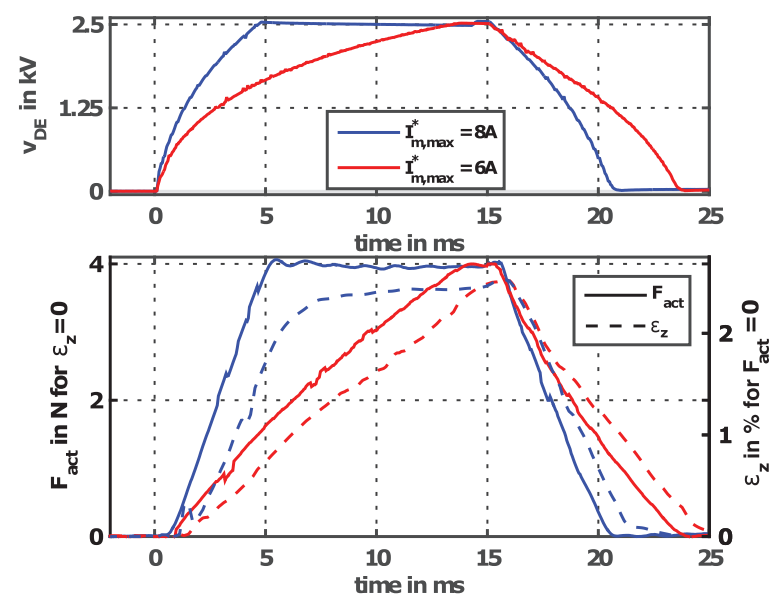

Figure I2. Open-loop behavior of the blocking force (solid lines) and no-load strain (dashed lines) of the DE stack-actuator when operating the flyback-converter with different peak currents $l_{m, \max }^{*}$.

utilized for the control only depends on the force $F_{a c t}^{*}$ and has not to be adapted.

Using two energy increments as hysteresis threshold by $n_{\delta s}=2$ according to equation (44) for the threepoint controller and choosing $I_{\min }=4 \mathrm{~A}, I_{\max }=8 \mathrm{~A}$, $S_{\min }=0.5 \mathrm{~mJ}$, and $S_{\max }=5 \mathrm{~mJ}$, respectively, for the adaption of the magnetizing current peak value $I_{m, \text { max }}^{*}$ in equation (45), the red dashed characteristics in Figure 13 are obtained. As can be seen in the second plot, immediately after the set-point step, the current is set to $I_{m, \max }^{*}=I_{\max }=8 \mathrm{~A}$, while during the transient response the current is reduced according to equation (45) until the peak value reaches its minimum $I_{\min }$ at steady state. By this adaption, both advantages - the higher dynamics with $I_{\max }$ and better accuracy with $I_{\min }$ - are achieved.

As illustrated in the bottom plot of Figure 13, the steady state is maintained by permanently switching between charging $\left(\bar{p} / \bar{p}_{\max }=1\right)$ and discharging $\left(\bar{p} / \bar{p}_{\max }=-1\right)$ in case of the two-point controllers. Here, the switching frequency equals almost the sample rate $f_{D S P}=20 \mathrm{kHz}$ of the real-time system on which the force control is implemented. The hysteresis as well as the off-state $\left(\bar{p} / \bar{p}_{\max }=0\right)$ enabled by the proposed three-point controller significantly reduce the switching 
frequency of the power converter by approximately $84 \%$ compared to the two-point controller with $I_{m, \max }^{*}=8 \mathrm{~A}$, without having a considerable impact on the control accuracy and dynamics.

Beside the adjustment of these constant set-points, a tracking application is investigated to determine the bandwidth of the force control. For this purpose, the small signal control behavior is characterized by applying a sine sweep set-point force with an offset of $3 \mathrm{~N}$ and an amplitude of $0.1 \mathrm{~N}$ as time varying reference value. The estimated magnitude and phase of the transfer function $F_{a c t} / F_{a c t}^{*}$ are depicted in the Bode plot in Figure 14. They are obtained using MATLAB ${ }^{\circledR}$ function tfestimate with the set-point force $F_{a c t}^{*}$ as input and the measured force $F_{a c t, m}$ as output. Up to the $3 \mathrm{~dB}$ cutoff frequency of approximately $500 \mathrm{~Hz}$, the force is accurately adjusted $\left(F_{a c t} / F_{a c t}^{*}=0 \mathrm{~dB}\right)$. Two resonance frequencies can be observed at about 700 and $900 \mathrm{~Hz}$, respectively, which are caused by mechanical eigenmodes of the test-rig. Compared to other (PID-based) control designs referenced in the introduction, for example, Sarban and Jones (2012), the bandwidth of the proposed controller is approximately one order of magnitude higher. Beside the fact that the SMC is predestined for the considered control plant, the bandwidth represents another big advantage of the proposed control enabling highly sophisticated applications, for example, as human-machine interface.

In a second scenario, a spring with stiffness $k_{\text {load }}=7.5 \mathrm{~N} / \mathrm{mm}$ is emulated by the position control of the linear motor, again. For this purpose, the setpoint position $\Delta z^{*}$ is determined depending on the desired set-point force $F_{a c t}^{*}$ by

$$
\Delta z^{*}=\frac{F_{\text {act }}^{*}}{k_{\text {load }}}
$$

Using the same parametrization for the three-point controller with hysteresis the measurement results depicted in Figure 15 are obtained. In addition to the measurement results, a comparison with the quantities estimated by the EKF is shown here. Different to the blocking-force scenario with $\varepsilon_{z}=0$ in Figure 13 now the DE stack-actuator deforms when a voltage is applied. This can be seen in the second subplot in Figure 15. Depending on the estimated strain $\hat{\varepsilon}_{z}$, the energy $U_{c, \text { diel }}^{*}$ is adapted according to equation (23) as can be seen by the corresponding voltage in the third subplot. Comparable to the previously investigated blocking-force scenario, the set-point force is adjusted in a few milliseconds. The dynamics of the strain are mainly caused by the dynamics of the utilized linear motor and its control to emulate the spring according to equation (49).

In Figure 16(a), the trajectory of the three state variables defined in equation (24) and according to Figure 8 is shown for the set-point step from $0 \mathrm{~N}$ to
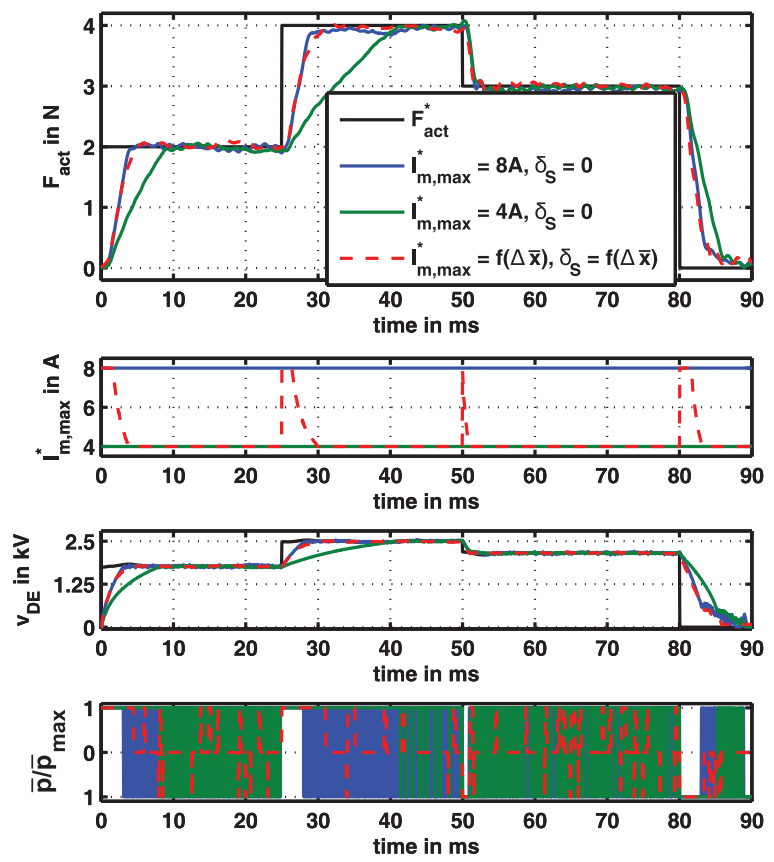

Figure 13. Different force adjustments for $\varepsilon_{z}=0$ using a twopoint controller with $l_{m, \max }^{*}=8 \mathrm{~A}$ (blue) and $I_{m, \max }^{*}=4 \mathrm{~A}$ (green) in comparison with the proposed three-point controller (red) with adaption of the peak current $l_{m, \max }^{*}=f(S(\Delta \overline{\mathbf{x}}))$ and hysteresis threshold $\delta_{S}=f(S(\Delta \overline{\mathbf{x}}))$, respectively.
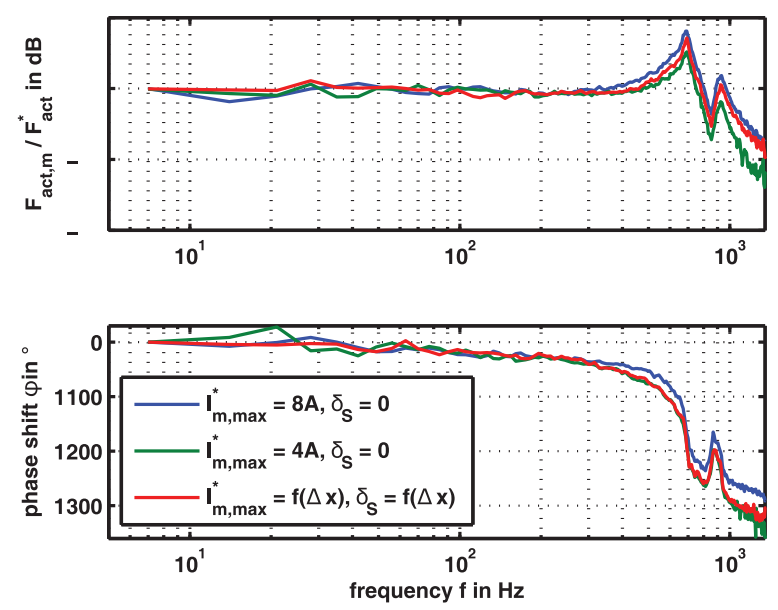

Figure 14. Bode plot of the small signal control behavior obtained from a sine sweep set-point force varying between $3 \mathrm{~N} \pm 0.1 \mathrm{~N}$ at constant strain $\varepsilon_{\mathrm{z}}=0$.

$F_{a c t}^{*}=1 \mathrm{~N}$. Starting from the initial control deviation $\mathbf{x}_{0}-\mathbf{x}^{*}$ during the reaching phase, the trajectory is first led to the switching surface and conducted toward the origin during the sliding mode, afterward. Both phases are clearly evident. Since the actuator is not activated initially, all entries in the initial state vector $x_{0}$ are zero. The deviations during the sliding mode are caused by the hysteresis of the three-point controller. As 

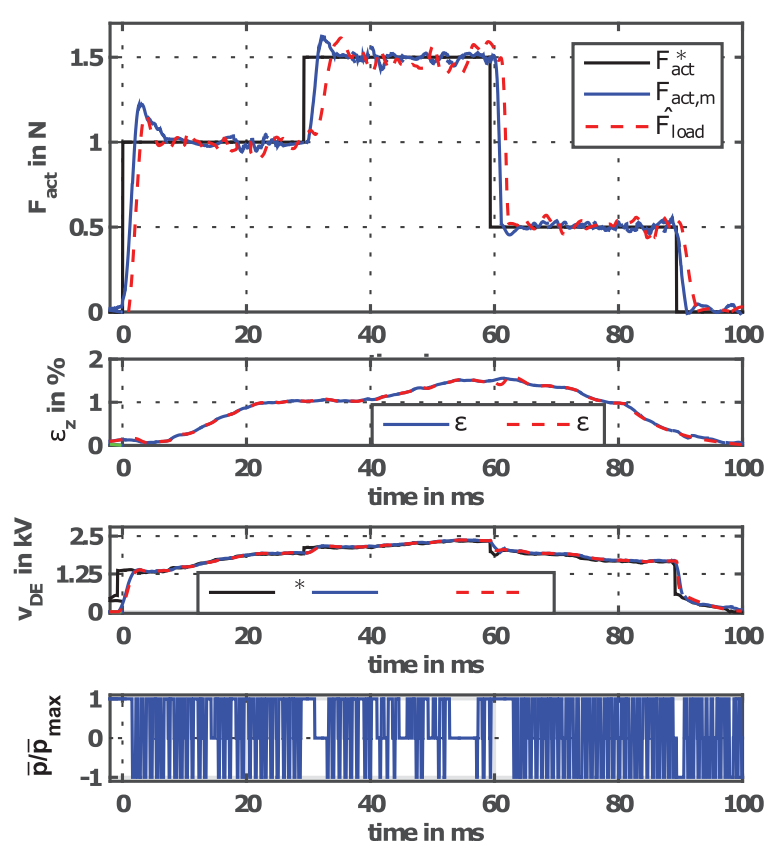

Figure I5. Comparison of measured and estimated quantities of the closed-loop behavior with the three-point force controller when operating against a spring load with a constant stiffness of $k_{\text {load }}=7.5 \mathrm{~N} / \mathrm{mm}$.

mentioned above and shown in the section of the sliding mode in Figure 16(b), the trajectory moves within the space $S(\Delta x)= \pm \delta_{S}$. However, the control hysteresis mainly affects $\dot{\varepsilon}_{z}$ and $\varepsilon_{k 1}$. The influence on the overall energy and the force $F_{a c t}$ is comparable small.

With the obtained measurement results, the feasibility and accuracy of the proposed sensorless force control have been illustrated. In this context, a further application of the control can be emphasized. In equation (23), a set-point energy $U_{c, \text { diel }}^{*}$ is defined to adjust an actuator force $F_{a c t}^{*}$. However, it is also possible to control the voltage $v_{p}$ across the DE capacitance $C_{p}$ by choosing

$$
U_{c, \text { diel }}^{*}=\frac{1}{2} \cdot C_{p}\left(\varepsilon_{z}\right) \cdot v_{p}^{* 2}
$$

Thus, with a simple modification of the set-point energy also the voltage can be controlled by the setpoint energy of the designed sliding mode control.

\section{Conclusion}

Multilayer DE stack-transducer are lightweight, scalable, and energy-efficient transducers that generate considerable tensile forces and deformations. To control their forces within this contribution, a force control was designed based on a holistic model combining the characteristic properties of the actuator and the driving power electronics. The utilized bidirectional flyback-

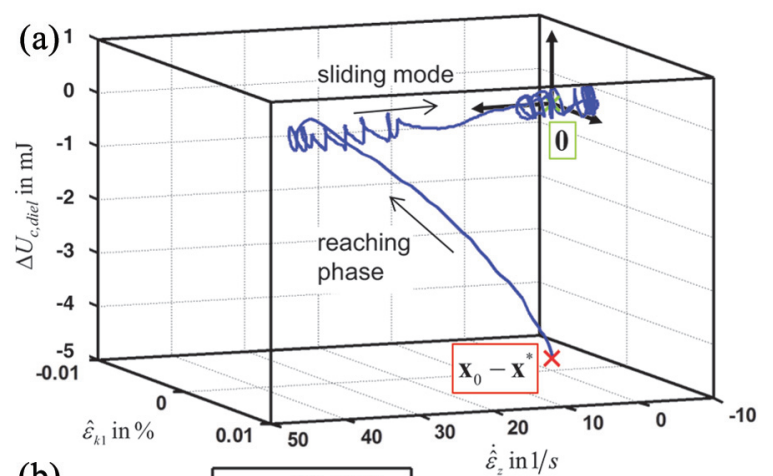

(b)

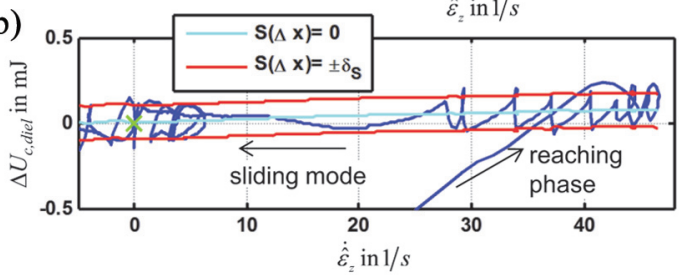

Figure 16. Trajectory of the SMC for the set-point step from $0 \mathrm{~N}$ to $F_{a c t}^{*}=I N(a)$ and detail of the sliding mode trajectory (b) when operating against the emulated spring.

converter supplies an almost constant feeding power that can be adapted by its inner current control. Considering this, extensions of the developed sliding mode force controller were introduced to ensure high dynamics during transient operation on one hand and good accuracy during steady state on the other hand by adapting the feeding power. Furthermore, using a three-point controller with hysteresis the switching frequency was significantly reduced by $84 \%$ without affecting the control quality.

To operate the force control, the electrical energy required to generate the desired transducer force has to be specified under consideration of the elastic deformation of the stack-actuator. Since the terminal voltage as well as the deformation of the stack-actuator are measured within our setup, a measurement of the force was not required for the force control. However, the setpoint energy has to be adapted continuously to compensate the current influence of the elastic deformation.

In a first setup, the developed sliding mode force control was validated by a blocking-force scenario where the deformation of the stack-actuator is prevented. Afterward, the linear motor of the utilized testrig was used to emulate a spring load in order to proof the force control quality in case of an arbitrary deformation. Both measurements showed good dynamics and accuracy of the proposed control as well as the benefits obtained by the extension, like reduced switching frequencies and improved accuracy.

Compared to other DE transducer controls referenced in the introduction, the main difference of the proposed one is that here a completely different electronic interface is used. While other research groups use a laboratory high-voltage amplifier, here the overall 
system comprising the DE actuator and the driving power electronics is modeled. For the overall system a tailored control is designed. The utilized bidirectional flyback-converter was specially designed for feeding DE transducer and is a promising topology for various commercial applications of DE actuators, for example, in automation technology. In particular, the proposed force control was designed using the constant output power provided by the converter as control interface. With this, an accurate and highly dynamic closed-loop operation is enabled that reaches steady state in just a few milliseconds. The overall system offers a wide range of highly sophisticated applications like active force feedback etc.

\section{Acknowledgement}

The authors would like to thank Marc Allan Clemens for his support regarding the implementation and experimental validation of the proposed force control.

\section{Declaration of conflicting interests}

The author(s) declared no potential conflicts of interest with respect to the research, authorship, and/or publication of this article.

\section{Funding}

The author(s) disclosed receipt of the following financial support for the research, authorship, and/or publication of this article: This contribution is accomplished within the project "Dielastar-Dielektrische Elastomere für Stellaktoren" (Dielectric Elastomer Actuators), funded by the Federal Ministry of Education and Research (BMBF) of Germany under grant number 13X4011E (see http://www.dielastar.de).

\section{ORCID iD}

Thorben Hoffstadt (iD https://orcid.org/0000-0003-2247-2271

\section{References}

Anderson IA, Gibsy TA, McKay TG, et al. (2012) Multi-functional dielectric elastomer artificial muscles for soft and smart machines. Journal of Applied Physics 112: 041101.

Biggs J, Danielmeier K, Hitzbleck J, et al. (2013) Electroactive polymers: developments of and perspectives for dielectric elastomers. Angewandte Chemie: International Edition in English 52(36): 9409-9421.

Chuc NH, Vuong NHL, Kim DS, et al. (2011) Fabrication and control of rectilinear artificial muscle actuator. IEEE/ ASME Transactions on Mechatronics 16(1): 167-176.

DeCarlo RA, Zak SH and Matthews GP (1988) Variable structure control of nonlinear multivariable systems: a tutorial. Proceedings of the IEEE 76(3): 212-232.

Dubois P, Rosset S, Niklaus M, et al. (2008) Voltage control of the resonance frequency of dielectric electroactive polymer (DEAP) membranes. Journal of Microelectromechanical Systems 17(5): 1072-1081.

Giousouf M and Kovacs G (2013) Dielectric elastomer actuators used for pneumatic valve technology. Smart Materials and Structures 22: 104010.

Graf C and Maas J (2011) Evaluation and optimization of energy harvesting cycles using dielectric elastomers. Proceedings of SPIE 7976: 1-16.

Gu GY, Gupta U, Zhu J, et al. (2015) Feedforward deformation control of a dielectric elastomer actuator based on a nonlinear dynamic model. Applied Physics Letters 107(4): 042907.

Hodgins M, York A and Seelecke S (2013) Experimental comparison of bias elements for out-of-plane DEAP actuator system. Smart Materials and Structures 22(9): 094016.

Hoffstadt T and Maas J (2015a) Analytical modeling and optimization of DEAP-based multilayer stack-transducers. Smart Materials and Structures 24(9): 094001.

Hoffstadt T and Maas J (2015b) Sensorless force control interface for DEAP stack-actuators. In: Proceedings of the ASME 2015 conference on smart materials, adaptive structures and intelligent systems, Colorado Springs, CO, 21-23 September, p. V001T03A023. New York: ASME.

Hoffstadt T and Maas J (2016) Sensorless BCM control for a bidirectional flyback-converter. In: Proceedings of the $42 n d$ annual conference of the IEEE industrial electronics society (IECON 2016), Florence, 23-26 October, p. 42. New York: IEEE.

Hoffstadt T and Maas J (2017) Adaptive sliding-mode position control for dielectric elastomer actuators. IEEE/ ASME Transactions on Mechatronics 22: 2241-2251.

Hoffstadt T, Griese M and Maas J (2014) Online identification algorithms for integrated dielectric electroactive polymer sensors and self-sensing concepts. Smart Materials and Structures 23(10): 104007.

Jones RW and Sarban R (2012) Inverse grey-box model-based control of a dielectric elastomer actuator. Smart Materials and Structures 21: 075019.

Kaal W and Herold S (2011) Electroactive polymer actuators in dynamic applications. IEEE/ASME Transactions on Mechatronics 16: 24-32.

Kalman RE (1960) Contributions to the theory of optimal control. Boletin de la Sociedad Matematica Mexicana 5: 102-119.

Kuhring S, Uhlenbusch D, Hoffstadt T, et al. (2015) Finite element analysis of multilayer DEAP stack-actuators. Proceedings of SPIE 9430: 94301L.

Maas J and Graf C (2011) Control concepts for dielectric elastomer actuators. Proceedings of SPIE 7976: $79761 \mathrm{H}$.

Maas J and Graf C (2012) Dielectric elastomers for hydro power harvesting. Smart Materials and Structures 21: 064006.

Maas J and Soetebier S (2012) Control concepts for smart actuators - review and prospects. In: Proceedings of the 13 th international conference on new actuators - ACTUATOR (ed. Borgmann H), Bremen, 18-20 June, vol. 12, pp. 270-275. Germany: WFB Wirtschaftsförderung Bremen $\mathrm{GmbH}$, Division Messe Bremen. 
Maas J, Tepel D and Hoffstadt T (2015) Actuator design and automated manufacturing process for DEAP-based multilayer stack-actuators. Meccanica 50: 2839-2854.

Maciejasz P, Eschweiler J, Gerlach-Hahn K, et al. (2014) A survey on robotic devices for upper limb rehabilitation. Journal of Neuroengineering and Rehabilitation 11: 3.

Pelrine R, Kornbluh R, Eckerle J, et al. (2001) Dielectric elastomers: generator mode fundamentals and applications. Proceedings of SPIE 4329: 148-156.

Piersol AG and Paez TL (2010) Harris' Shock and Vibration Handbook (McGraw-Hill handbooks). 6th ed. New York: McGraw-Hill.

Price AD and Ask A (2014) Integrated design optimization of dielectric elastomer actuators in high-performance switchgear. In: Proceedings of the ASME conference on smart materials, adaptive structures and intelligent systems (SMASIS), Newport, RI, 8-10 September, paper no. SMASIS2014-7574. New York: ASME.

Rizzello G, Naso D, York A, et al. (2016) Closed loop control of dielectric elastomer actuators based on self-sensing displacement feedback. Smart Materials and Structures 25(3): 035034.

Rosset S, Araromi OA, Schlatter S, et al. (2016) Fabrication process of silicone-based dielectric elastomer actuators. Journal of Visualized Experiments 108: e53423.

Sarban R and Jones RW (2012) Physical model-based active vibration control using a dielectric elastomer actuator. Journal of Intelligent Material Systems and Structures 23(4): 473-483.
Sokal NO and Redl R (1997) Control algorithms and circuit designs for optimal flyback-charging of an energy-storage capacitor (e.g., for flash lamp or defibrillator). IEEE Transactions on Power Electronics 12: 885-894.

Tomori H, Midorikawa Y and Nakamura T (2013) Vibration control of an artificial muscle manipulator with a magnetorheological fluid brake. Journal of Physics: Conference Series 412: 012053.

Venkatesan K (1989) Current mode controlled bidirectional flyback converter. In: Proceedings of the 20th annual IEEE power electronics specialists conference (PESC '89 record), Milwaukee, WI, 26-29 June, pp. 835-842. New York: IEEE.

Welch G and Bishop G (2001) An Introduction to the Kalman Filter. Chapel Hill, NC: University of North Carolina at Chapel Hill.

Wilson ED, Assaf T, Pearson MJ, et al. (2016) Cerebellarinspired algorithm for adaptive control of nonlinear dielectric elastomer-based artificial muscle. Journal of the Royal Society Interface 13(122): 20160547.

Zhang R, Lochmatter P, Kunz A, et al. (2006) Spring roll dielectric elastomer actuators for a portable force feedback glove. Proceedings of SPIE 6168: 61681T.

Zou J, Gu GY and Zhu LM (2016) Open-loop control of creep and vibration in dielectric elastomer actuators with phenomenological models. IEEE/ASME Transactions on Mechatronics 22(1): 51-58. 